\title{
PAPER
}

\section{Efficacy of methylprednisolone pulse therapy on neuroleptic malignant syndrome in Parkinson's disease}

\author{
Y Sato, T Asoh, N Metoki, K Satoh
}

J Neurol Neurosurg Psychiatry 2003;74:574-576

See end of article for authors' affiliations

......................

Correspondence to:

Dr Y Sato, Department of

Rehabilitation Medicine,

Institute of Brain Science,

Hirosaki University School

of Medicine, 5 Zaifu-cho,

Hirosaki 036-8562, Japan;

noukenrs@cc.hirosaki-u.ac.jp

Received

7 October 2002

Accepted

11 January 2003
Background: Neuroleptic malignant syndrome (NMS) is a dangerous complication in patients with Parkinson's disease (PD).

Aims: To evaluate the efficacy of methylprednisolone pulse therapy compared to placebo in PD patients with NMS.

Methods: In a double blind, placebo controlled study, 20 PD patients with NMS received steroid pulse therapy for three days, and 20 PD patients received placebo. Both groups received levodopa, bromocriptine, and dantrolene.

Results: NMS in the steroid group healed within 10 days in 17 patients; median value of duration of illness of NMS in this group was 7 days (range 4-20). NMS in the placebo group healed within 10 days in five patients; in the remaining 15, it persisted for 12-27 days after the onset of NMS; median value of duration illness of NMS in this group was 18 days. Hyperthermia, rigidity, and consciousness improved within 10 days in many patients in the steroid group; these signs persisted more than 10 days in many patients in the placebo group.

Conclusions: Steroid pulse therapy is useful in NMS for reducing the illness duration and improving symptoms.
$\mathrm{N}$ euroleptic malignant syndrome (NMS) is a dangerous, often fatal, complication following withdrawal of antiparkinsonian agents in patients with Parkinson's disease (PD). ${ }^{1-3}$ Complications affect almost all organ systems. Cardinal features include hyperthermia, muscular rigidity, altered consciousness, autonomic dysfunction, and an increased serum level of creatine kinase (CK). ${ }^{12}$ Treatment is largely supportive, and levodopa ${ }^{4}$ and dopamine agonists, such as bromocriptine, ${ }^{5}$ have been used with only slight improvement in clinical outcome. NMS sometimes has a prolonged clinical course, persisting for more than a few weeks despite the above therapy.

We have been been aware that methylprednisolone pulse therapy appears to be effective in NMS of PD for improving its clinical manifestations and shortening duration of illness in clinical practice. We therefore conducted a randomised trial to evaluate the efficacy of methylprednisolone pulse therapy compared to a placebo in NMS patients with PD.

\section{PATIENTS AND METHODS}

We studied 40 patients identified with their first episode of NMS in the course of PD. Patients who had been admitted to the Futase Social Insurance Hospital between January 1995 and December 1997 on the day of NMS onset were enrolled. The diagnosis of NMS was made according to the criteria of Susman $^{6}$ based on the four major signs-hyperthermia, muscular rigidity, changes in mental status, and autonomic dysfunction. We repeatedly monitored haematological and blood chemical data, including serum CK.

The study was approved by the local ethics committee, and informed consent was obtained from all study subjects in the presence of a witness.

The patients were assigned to one of the two study groups by means of computer generated random numbers. Patients received $1000 \mathrm{mg}$ of intravenous methylprednisolone (SoluMedrol, Upjohn Pharmaceuticals, Osaka, Japan) $(\mathrm{n}=20)$ or a placebo $(n=20)$. Methylprednisolone or placebo was administered on the day of onset of NMS and was continued for two days. In both groups, all patients received oral levodopa (300 $\mathrm{mg} /$ day) and bromocriptine $(7.5 \mathrm{mg} /$ day) from the day of onset of NMS until NMS was clinically healed. In addition, dantrolene sodium ( $75 \mathrm{mg} /$ day) was administered by intravenous infusion on the day of onset of NMS and continued daily until healing of NMS was evident in both groups. If the body temperature exceeded $39^{\circ} \mathrm{C}$, cooling was performed. Two patients in each group developed pneumonia which was treated by antibiotics; the other patients did not receive antibiotic therapy. All patients were managed in the intensive care unit; a general medical evaluation was performed and laboratory values were assessed on entry into the study to obtain baseline values, and again after 10 days.

All statistical procedures were performed using the Statview J 5.0 software packages (Abacus Concepts, Berkeley, CA). Data are presented as mean (SD). Group differences of the categorical data were tested by $\chi^{2}$ analyses. The unpaired $t$ test was used to assess the significance of the differences of variables between the methylprednisolone or placebo group. Vital signs and individual laboratory values were computed and expressed as percentage change from baseline. The two groups were compared with respect to vital signs and the laboratory values using the Wilcoxon rank sum test. Probability values less than $5 \%$ were considered statistically significant.

\section{RESULTS}

Demographic and baseline clinical characteristics of PD patients at study entry

Table 1 presents the demographic and baseline clinical features of the two patient groups in the study. At baseline, there were no significant differences in age, gender, illness

Abbreviations: CK, creatine kinase; DIC, disseminated intravascular coagulation; NMS, neuroleptic malignant syndrome; PD, Parkinson's disease 
Table 1 Demographic and baseline clinical characteristics of the Parkinson's disease patients at study entry

\begin{tabular}{|c|c|c|c|}
\hline Variables & Placebo $(n=20)$ & Steroid $(n=20)$ & $p$ value* \\
\hline Age, years & $72.4 \pm 3.9$ & $73.6 \pm 4.3$ & 0.34 \\
\hline Gender, M/F & $11 / 9$ & $10 / 10$ & NSt \\
\hline Duration of illness, years & $8.1 \pm 1.8$ & $8.2 \pm 2.1$ & 0.81 \\
\hline \multicolumn{4}{|l|}{ Antiparkinsonian drugs } \\
\hline Levodopa/caridpopa or levodopa/benserazide & 20 & 19 & \\
\hline Amantadine & 8 & 9 & \\
\hline Bromocriptine & 12 & 13 & \\
\hline Trihexyphenidyl & 6 & 7 & $0.98 \dagger$ \\
\hline \multicolumn{4}{|l|}{ Reason for discontinuing antiparkinsonian drugs } \\
\hline Psychiatric symptoms & 6 & 5 & \\
\hline Dyskinesia & 7 & 8 & \\
\hline On-off & 3 & 4 & \\
\hline Diarrhoea & 2 & 2 & \\
\hline None & 2 & 1 & $0.96 \dagger$ \\
\hline Heart rate (beats/min) & $127 \pm 13$ & $128 \pm 15$ & 0.74 \\
\hline Systolic blood pressure $(\mathrm{mm} \mathrm{Hg})$ & $93 \pm 9$ & $91 \pm 14$ & 0.61 \\
\hline Diastolic blood pressure $(\mathrm{mm} \mathrm{Hg})$ & $54 \pm 7$ & $56 \pm 12$ & 0.60 \\
\hline Body temperature $\left({ }^{\circ} \mathrm{C}\right)$ & $39.8 \pm 0.7$ & $39.6 \pm 0.7$ & 0.37 \\
\hline Serum creatine kinase (IU) & $4904 \pm 2406$ & $4143 \pm 2204$ & 0.31 \\
\hline White blood cell count (per $\mathrm{mm}^{3}$ ) & $17392 \pm 4789$ & $16210 \pm 4825$ & 0.44 \\
\hline
\end{tabular}

duration of PD, antiparkinsonian drugs, or reason for discontinuing of antiparkinsonian drugs. At baseline, all patients in the two PD groups had hyperthermia, tachycardia, hypotension, rigidity, altered consciousness, high serum concentrations of CK, and leucocytosis in the peripheral blood. All patients were extremely ill, bedridden, and immobile.

\section{Clinical course and changes in variables}

NMS in methylprednisolone group healed within 10 days in 17 patients, while NMS in the remaining three patients persisted between 13 and 20 days after the onset (see table 2). Thus, median value of duration of illness of NMS in this group was 7 days (range 4-20). Disseminated intravascular coagulation (DIC) occurred in three patients in the placebo group; one died within 10 days. In the methylprednisolone group, there were no complications resulting in death. NMS in the placebo group healed within 10 days in five patients, while NMS in the remaining 15 patients persisted between 12 and 27 days after the onset of NMS. Thus, median value of duration illness of NMS in this group was 18 days (range 5-27). The number of patients who healed 10 days after the onset of NMS was significantly higher in the methylprednisolone group than the placebo group ( $\mathrm{p}<0.0001 ; \chi^{2}$ analyses).

During the 10 day study period, heart rate decreased significantly more in the methylprednisolone group than in the placebo group $(p=0.0027$; Wilcoxon rank sum test). Heart rate normalised in 18 patients in the methylprednisolone group, and in six patients in the placebo group. With the exception of patients who died, systolic and diastolic blood pressure increased in both groups during the 10 day study period, and systolic blood pressure in the methylprednisolone group was increased significantly more than in the placebo group $(\mathrm{p}=0.0014)$. Systolic and diastolic blood pressure normalised in 18 patients in the methylprednisolone group during or just after the administration of steroid, while blood pressure normalised in 12 patients in the placebo group 10 days after baseline. Body temperature decreased significantly more in the methylprednisolone group than in the placebo group $(p=0.0027)$. Body temperature normalised in all patients in the methylprednisolone group during or just after administration of steroid, while it normalised in nine patients in the placebo group 3-9 days after onset. Rigidity improved within 10 days in 19 patients in the methylprednisolone group. It improved in 12 patients within 10 days, and persisted between 12 and 21 days in the remaining six patients in the placebo group. Altered consciousness became clear in 19 patients in the methylprednisolone group within 10 days, while it became to clear within 10 days in 15 patients; it persisted between 11 and 14 days in the placebo group. Serum CK in the methylprednisolone group declined significantly compared to the placebo group ( $\mathrm{p}<0.0001)$; CK normalised in all 20 patients in the methylprednisolone group and in six patients in the placebo group at this point. The white blood cell count in the methylprednisolone group decreased significantly compared to the placebo group ( $\mathrm{p}<0.0326)$.

\section{DISCUSSION}

In the present study, methylprednisolone shortened the illness duration of NMS in PD and improved signs of NMS and laboratory values within 10 days. On the contrary, abnormal signs

Table 2 Selected changes after 10 days in the 40 subjects

\begin{tabular}{lllr}
\hline & \multicolumn{2}{l}{ Percentage change after 10 days follow up (result at 10 days) } & \\
\cline { 2 - 3 } & \multicolumn{1}{l}{ Placebo $(\mathrm{n}=18)$} & Steroid $(\mathrm{n}=20)$ & p value* \\
\hline Heart rate (beats/min) & $-4.3 \pm 1.9(80 \pm 16)$ & $-6.5 \pm 2.0(68 \pm 5)$ & 0.0027 \\
Systolic blood pressure $(\mathrm{mm} \mathrm{Hg})$ & $+20.9 \pm 18.1(110 \pm 9)$ & $+33.4 \pm 13.5(122 \pm 7)$ & 0.0114 \\
Diastolic blood pressure $(\mathrm{mm} \mathrm{Hg})$ & $+22.5 \pm 19.3(64 \pm 7)$ & $+28.7 \pm 19.9(68 \pm 5)$ & 0.13 \\
Body temperature $\left({ }^{\circ} \mathrm{C}\right)$ & $-4.3 \pm 1.9(37.5 \pm 0.5)$ & $-7.5 \pm 2.3(36.1 \pm 0.6)$ & 0.0001 \\
Serum creatine kinase $(\mathrm{IU})$ & $-83.3 \pm 12.2(631 \pm 316)$ & $-98.8 \pm 1.5(56 \pm 17)$ & $<0.0001$ \\
White blood cell count $\left(\right.$ per $\left.\mathrm{mm}^{3}\right)$ & $-44.2 \pm 10.8(9451 \pm 2226)$ & $-52.2 \pm 15.6(7339 \pm 1946)$ & 0.0326 \\
\hline Results expressed as mean $\pm \mathrm{SD}$. & & & \\
*Wilcoxon rank sum test. & & & \\
\end{tabular}


and laboratory values in the placebo group persisted for longer than in the methylprednisolone group. NMS is a highly fatal condition but we only had a single fatal case in the placebo group. All patients were admitted on the day of NMS development for intensive care management, and appropriate specific therapy was initiated immediately. Combination therapy with dantrolene is effective in reducing mortality of NMS. ${ }^{7-9}$ Tsutumi and colleagues ${ }^{8}$ reported 21 cases of NMS complicated psychiatric disorders treated with dantrolene; the results were remarkable with no fatalities. Dantrolene may be also useful in lowering mortality in PD patients with NMS, and inclusion of methylprednisolone may be of benefit to reduce the severity of the condition.

The precise mechanism of the action of methylprednisolone in NMS is unknown. Hyperthermia is the central feature of NMS, but its aetiology remains elusive. Two competing hypotheses implicate either hypothalamic dysfunction (inappropriate "set point") or direct myotoxicity (excessive peripheral heat production).. ${ }^{10}$ Rat ventral tegmental area and adjacent substance nigra have been shown to possess a moderate number of cells with active glucocorticoid receptors. In addition, several observations have suggested that glucocorticoids might contribute to an increase in dopaminergic activity. Glucocorticoids have experimentally been shown to have state dependent stimulant effects on mesencephalic dopaminergic transmission, and an interaction between these two factors might be involved in the appearance of behavioural disturbances. ${ }^{11}$ Thus, it is postulated that methylprednisolone enhances dopaminergic activity in NMS in PD, although its stabilising effect on lysosomal membrane is also likely to account for ameliorating the organ system manifestation of NMS.

\section{Authors' affiliations}

Y Sato, T Asoh, Department of Neurology, Futase Social Insurance Hospital, lizuka 820-0054, Japan

N Metoki, Department of Rehabilitation Medicine, Institute of Brain Science, Hirosaki University School of Medicine, Hirosaki 036-8562, Japan

K Satoh, Department of Vascular Biology, Institute of Brain Science, Hirosaki University School of Medicine, Hirosaki 036-8562, Japan

\section{REFERENCES}

1 Henderson VW, Wooten GF. Neuroleptic malignant syndrome: a pathogenetic role for dopamine receptor blockade? Neurology 1981:21:132-7.

2 Sechi GP, Tanda F, Mutani R. Fatal hyperpyrexia after withdrawal of levodopa. Neurology 1981;24:245-51.

3 Gibb WRG, Griffith DNW. Levodopa withdrawal syndrome identical to neuroleptic malignant syndrome. Postgrad Med J 1986;62:59-60.

4 Nisijima K, Noguchi M, Ishiguro T. Intravenous injection of levodopa is more effective than dantrolene as therapy for neuroleptic malignant syndrome. Biol Psychiatry 1997;41:913-14

5 Rossenberg MR, Green M. Neuroleptic malignant syndrome: review of response to therapy. Arch Intern Med 1989;149:1927-31.

6 Susman VL. Clinical management of neuroleptic malignant syndrome. Psychiatr Q 2001;72:25-36.

7 Tsujimoto S, Maeda K, Sugiyama T, et al. Efficacy of prolonged large-dose dantrolene for severe neuroleptic malignant syndrome. Anesth Analg 1998:86:1143-4.

8 Tsutsumi Y, Yamamoto K, Matsuyama S, et al. The treatment of neuroleptic malignant syndrome using dantrolene sodium. Psychiatry Clin Neurosci 1998;52:433-8

9 Sakkas P, Davis JM, Hua J, et al. Pharmacotherapy of neuroleptic malignant syndrome. Psych Annals 1991:21:157-64.

10 Czyrak A, Chocyk A. Search for the presence of glucocorticoid receptors in dopaminergic neurons of rat ventral tegmental area and substantia nigra. Pol J Pharmacol 2001;53:681-94

11 Piazza PV, Rouge-Pont F, Deroche V, et al. Glucocorticoids have state-dependent stimulant effects on the mesencephalic dopaminergic transmission. Proc Natl Acad Sci U S A 1996;93:8716-20. 


\section{Retraction}

Y Sato, T Asoh, N Metoki et al. Authors' reply. J Neurol Neurosurg Psychiatry 2004;75:511 ret.

We are retracting this letter in light of the retraction of the article to which it relates.

J Neurol Neurosurg Psychiatry 2018;89:e3. doi:10.1136/jnnp.74.5.0574ret

(A) Check for updates 


\section{Retraction}

Sato Y, Asoh T, Metoki N, et al. Efficacy of methylprednisolone pulse therapy on neuroleptic malignant syndrome in Parkinson's disease. J Neurol Neurosurg Psychiatry 2003;74:574-576.

This article has been retracted. The editor received a letter from Dr Yoshihiro Sato retracting this article and two others also published in Journal of Neurology Neurosurgery and Psychiatry. Dr Sato states the reason for retraction as 'due to scientific misconduct. All co-authors did not participate study design, data collection, data analysis, interpretation of data and drafting manuscripts in these 3 articles. Thus, all co-authors are honorary.'

Despite repeated requests for further explanation, we have not received any response from Dr Sato's institution.

It was brought to our attention that several other journals have retracted articles by Dr Sato. Neurology published an article 'Systematic review and statistical analysis of the integrity of 33 randomized controlled trials' by Boland $e t \mathrm{al}^{1}$ indicating that the data in those trials were not reliable. The data supplement for the article in Neurology lists the three papers published in Journal of Neurology Neurosurgery and Psychiatry providing evidence that the reported findings in those articles are unreliable.

J Neurol Neurosurg Psychiatry 2018;89:e9. doi:10.1136/jnnp.74.5.574ret

\section{Check for updates}

\section{REFERENCE}

1 Bolland MJ, Avenell A, Gamble GD, et al. Systematic review and statistical analysis of the integrity of 33 randomized controlled trials. Neurology 2016;87:2391-402. 